\title{
Chemi-Structural Stabilization of Formamidinium Lead lodide Perovskite by Using Embedded Quantum Dots
}

Sofia Masi, ${ }^{[a] *}$ Carlos Echeverría-Arrondo, ${ }^{[a]}$ K. M. Muhammed Salim, ${ }^{[a]}$ Thi Tuyen Ngo, ${ }^{[a]}$ P. F. Mendez, ${ }^{[a, b]}$ Eduardo López-Fraguas, ${ }^{[a, c]}$ David F. Macias-Pinilla, ${ }^{[a, d]}$ Josep Planelles ${ }^{[d]}$, Juan I. Climente $^{[d]^{*}}$ and Iván Mora-Seró ${ }^{[a]^{*}}$

[a] Institute of Advanced Materials (INAM), Universitat Jaume I, Av. Sos Baynat, s/n, 12071 Castelló, Spain

[b] Facultad de Ciencias Químico Biológicas, Universidad Autónoma de Sinaloa, Cd. Universitaria, Av. de las Américas y Josefa Ortiz S/N, 80000, Culiacán, Sinaloa, México

[c] Electronic Technology Department, Universidad Carlos III de Madrid (GDAF-UC3M), Leganés, 28911, Madrid, Spain

[d] Departament de Química Física i Analítica, Universitat Jaume l, Av. Sos Baynat s/n, 12071, Castelló, Spain

Corresponding Authors: masi@uji.es; climente@uji.es; sero@uji.es

\section{Abstract}

The approaches to stabilize the perovskite structure of formamidinium lead iodide (FAPI) commonly result in a blue-shift of the band gap, which limits the maximum photo-conversion efficiency. Here, we report the use of PbS colloidal quantum dots (QDs) as stabilizing agent, preserving the original low band gap of $1.5 \mathrm{eV}$. The surface chemistry of PbS plays a pivotal role, by developing strong bonds with the black phase but weak ones with the yellow phase. As a result, stable perovskite FAPI black phase can be formed at temperatures as low as $85^{\circ} \mathrm{C}$ in just 10 minutes, setting a record of concomitantly fast and low temperature formation for FAPI, with important consequences for industrialization. FAPI thin films obtained through this procedure reach an open circuit potential $\left(V_{o c}\right)$ of $1.105 \mathrm{~V}-91 \%$ of the maximum theoretical $\mathrm{V}_{\mathrm{oc}}$ - and preserve the efficiency for more than 700 hours. These findings reveal the potential of strategies exploiting the chemi-structural properties of external additives to relax the tolerance factor and optimize the optoelectronic performance of perovskite materials. 
Production expenses and photoconversion efficiency (PCE) are critical factors impacting on the final cost of the solar energy technologies. Perovskite solar cells (PSCs) bring promise for affordable and efficient devices, with PCE recently reaching $25.2 \% .^{1}$ Beyond excellent optoelectronic properties, this newcomer family of materials has a great composition versatility, that enables the synthesis of perovskites with tunable bandgap, ${ }^{2}$ producing a broad range of possibilities for light emitting systems. For the development of photovoltaic systems, however, the range of material choice is confined to cations suitable for 3D perovskites with good charge transport properties, namely methylammonium (MA), formamidinium (FA) and Cs. ${ }^{3}$ Moreover, for a single layer absorber, a band gap as close as possible to $1.4 \mathrm{eV}$ is required, as then the ShockleyQueisser model predicts a maximum theoretical efficiency of $33 \%{ }^{4}$ FAPI perovskite phase has the lowest band gap, ${ }^{5} 1.48 \mathrm{eV},{ }^{6,7}$ with a maximal theoretical PCE (PCE $\max$ ) of $32.3 \%{ }^{8}$ With the further advantage of higher thermal stability in comparison to MA-based PSCs, FAPI perovskite crystals fulfill an ideal compromise between a fully inorganic and a hybrid perovskite in terms of both thermal stability ${ }^{9}$ and band gap. ${ }^{6}$

The structural stability is however an issue. FAPI has a Goldschmidt tolerance factor close to unity, but its polymorphism limits the stability of the black phase -which includes cubic $(\alpha)$ and tetragonal ( $\beta$ and $\gamma$ ) phases- ${ }^{3}, 10-13$ in favor of the yellow -hexagonal $(\delta)$ - phase, ${ }^{14}$ which is photoinactive with a band gap of $2.43 \mathrm{eV} .{ }^{15}$ Consequently, the black phase is the most stable phase for temperatures over $165{ }^{\circ} \mathrm{C}$ only. ${ }^{10}$ This implies it must be synthesized at high temperatures (the annealing range is $150-185^{\circ} \mathrm{C}$ for $30 \mathrm{~min}$ ), ${ }^{16-18}$ and after cooling down to room temperature it constitutes a metastable phase that transforms into the yellow phase in a relatively short time, especially in humid environments. ${ }^{19}$ Cation alloying is a standard technique to decrease the Goldschmidt effective tolerance factor. ${ }^{10}$ Different studies report the addition of methylammonium $\left(\mathrm{MA}^{+}\right),{ }^{20}$ cesium $\left(\mathrm{Cs}^{+}\right),{ }^{12,}{ }^{21-23}$ potassium $\left(\mathrm{K}^{+}\right),{ }^{24}$ rubidium $\left(\mathrm{Rb}^{+}\right),{ }^{25}$ or large organic cations forming 2D perovskites ${ }^{26-28}$ to stabilize the mixed cation perovskite $\alpha$-phase ${ }^{29,30}$ with the double advantage of a higher reproducibility and the formation of the $\alpha$-phase at lower temperature $\left(100-150{ }^{\circ} \mathrm{C}\right)$. Yet, in the case of Pb-perovskites, smaller A-site cation causes octahedral tilting resulting in an increased band gap. ${ }^{31,32}$ This is an undesired side-effect, as it shifts the gap energy away from the optimal value in terms of $\mathrm{PCE}_{\max },{ }^{4,8}$ in most of the cases, For 
example, the extensibility used $\mathrm{Cs}_{0.05} \mathrm{MA}_{0.16} \mathrm{FA}_{0.79} \mathrm{~Pb}\left(\mathrm{I}_{0.83} \mathrm{Br}_{0.17}\right)_{3}$ presents a band gap of $1.63 \mathrm{eV},{ }^{12}$ limiting $P C E_{\max }$ to $29.8 \%{ }^{8}$ Recent research efforts are trying to reduce the perovskite band gap by removing $\mathrm{Br}^{20,33}$ This strategy has yielded the current PCE published record of $23.3 \%{ }^{20}$ with the reduction of band gap limited to $1.55 \mathrm{eV}$, as $8 \%$ of MA must be added to stabilize FAPI black phase. The use of Cs instead of MA to stabilize the $\alpha$-phase in RbCsFAPbl 3 succeeded in further reducing the band gap to $1.53 \mathrm{eV}$, where $\mathrm{Rb}$ has the additional role of increasing the reproducibility. ${ }^{33}$ However, very recently FAPI stabilization with high performances has been obtained recently adding ammonium valeric $\operatorname{acid}^{28}$ and methylenediammonium dichloride ${ }^{34}$ as additives, with no significant blue-shift of the FAPI gap.

In this work, we propose to stabilize FAPI perovskite not through the introduction of alternative cations or anions to $\mathrm{FA}^{+}$and $\mathrm{I}^{-}$, but by taking benefit of the synergistic interaction of halide perovskites with colloidal PbS quantum dots (QDs). ${ }^{35}$ MA-based halide perovskite thin films with embedded $\mathrm{QDs}^{36}, 37$ have been employed for the development of LEDs, $^{38}$ photodetectors ${ }^{39}$ and solar cells. ${ }^{40}$ Inorganic $\mathrm{CsPb}\left(\mathrm{I}_{1-\mathrm{x}} \mathrm{Br} \mathrm{r}_{\mathrm{x}}\right)_{3}$ perovskites with embedded PbS QDs, due to the zero-mismatch, increase the stability (photoluminescence $(\mathrm{PL}$ ) reduction of just $10 \%$ after an annealing of one hour while for samples without embedded QDs the PL was reduced in a $50 \%$ ), especially for $\mathrm{Br}$ rich perovskite with the same lattice parameter than $\mathrm{PbS} .{ }^{41}$ We show that the use of embedded PbS QDs presents multiple advantages over alloying, since it stabilizes the black phase in ambient conditions, while preserving the narrow band gap of pure FAPI. Also, the synthetic conditions become milder, with temperatures as low as $85^{\circ} \mathrm{C}$, annealing times of only 10 minutes and no deleterious effect on the thermal stability of the resulting film. PSCs prepared out of FAPI films stabilized with PbS QDs demonstrated higher performance and reproducibility than their counterparts without QDs, as well as a high open circuit potential, $V_{o c}$, of $1.105 \mathrm{~V}$ for pure FAPI. PSCs with embedded PbS QDs further exhibited a significant improvement in long term stability, with only $16 \%$ decrease of the initial PCE after 720 hours, as compared to QD free samples, which presented a reduction in efficiency of $30 \%$ after a single day since preparation, and no performance at all after 500 hours. To elucidate the physical processes involved in the superior stabilization of FAPI by means PbS QDs, we combined systematic studies on the effect of QD size and concentration with a two-level theoretical analysis based on Density 
Functional Theory (DFT) and linear elastic theory of continuous media. The PbS-FAPI interface is found reduce the thermodynamic preference for the yellow phase, by creating stronger chemical bonds with the black one, and to propagate strain fields over long distances, which favor the formation of large grain sizes. These factors point to surface chemistry engineering as a less invasive and yet efficient methodology to stabilize halide perovskite materials.

The first step carried out for the incorporation of the inorganic PbS QDs into the FAPI perovskite matrix is to make both materials chemically compatible in solution for the spin coating deposition. The finest method is to replace the organic ligand, specifically the oleic acid (OA), coming from the synthesis of the QDs, with a new one. In this case, the ligand has to anchor itself to the surface of the PbS, avoiding their aggregation in solution, but at the same time it has to be polar, to guarantee the solubility of the quantum dots in the perovskite solvent, namely dimethylsulfoxide (DMSO) or dimethylformammide (DMF). The ligand exchange procedure used is similar to previously reported in literature, ${ }^{36,37,42}$ substituting the organic capping of PbS QDs by a FAPI ligand, see details in the Experimental Section. After the ligand exchange PbS/FAPI QDs are soluble in DMF solution, see Figure 1a. A Fourier-Transform Infrared Spectroscopy (FTIR) measurement of the dried PbS QDs, and deposited in thin film, before the ligand exchange, exhibits the signals related to the oleic acid, the stretching modes of the $\mathrm{O}-\mathrm{H}$ at $3,446 \mathrm{~cm}^{-1}$, of the $\mathrm{C}-\mathrm{H}$ at $3,020-2,800 \mathrm{~cm}^{-1}$ and of the carboxyl $\mathrm{C}=\mathrm{O}$ at $1,527 \mathrm{~cm}^{-1}$. These modes are totally absent after ligand exchange, see Figure $1 b$, pointing the success of the ligand exchange procedure. Furthermore, the solutions of the PbS/FAPI in DMSO or DMF are stable until the concentration of $50 \mathrm{mg} / \mathrm{ml}$, indicating the universality and robustness of the method, which in fact preserves the features of the as synthesized PbS QDs, Figure 1c, after the ligand exchange, see Figure 1d.

Transmission Electron Microscopy (TEM) images reveal an increase of the average size of QDs comparing the as-synthesized OA capped PbS QDs (PbS/OA) and FAPI capped PbS/FAPI QDs, see Figure $1 \mathrm{c}$ d. The statistic distribution is plotted in Figure S1 in good agreement with the estimated QDs size from PL spectra, see Figure $2 \mathrm{a}$, around $3.8 \pm 1.8 \mathrm{~nm}$ and $4.7 \pm 1.5 \mathrm{~nm}$ for the $\mathrm{PbS} / \mathrm{OA}$ and the PbS/FAPI, respectively. ${ }^{43}$ As synthesized PbS/OA QDs present cubic rock salt structure, see Figure 1e. After ligand exchange and annealing a shell of FAPI is formed ${ }^{44}$ with 
majority black phase, despite a small contribution from yellow phase is visible in the X-Ray Diffraction (XRD) pattern, see Figure $1 \mathrm{f}$.

a)
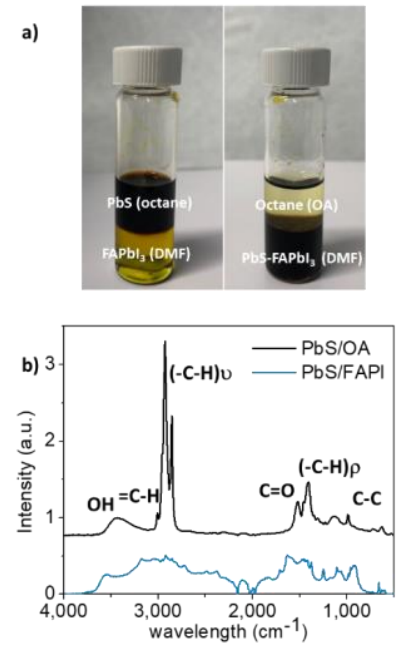
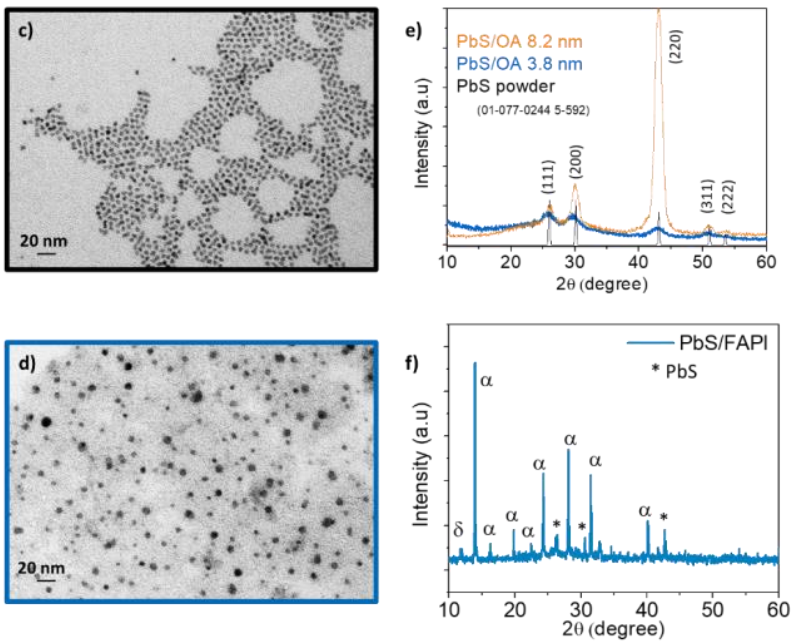

Figure 1. (a) Pictures (left) before the ligand exchange with the PbS QDs capped with oleic acid in octane solution and FAPI precursors ( $\mathrm{Pbl}_{2}$ and FAI) in DMF and (right) after the ligand exchange with PbS/FAPI QDs in DMF solution. (b) FTIR of the PbS QDs before and after the ligand exchange. TEM images of $10 \mathrm{mg} / \mathrm{ml} \mathrm{PbS}$ QDs solutions before and after ligand exchange; (c) with the oleic acid and (d) with FAPI ligands, respectively; (e) XRD of the oleic acid capped PbS QDs spin coated on glass from octane solution and (f), XRD of PbS/FAPI QDs spin coated and annealed from DMF solution.

Ligand exchange produces a change in the photoluminescence (PL) emission peak (Figure 2a) and in the absorbance of the PbS QDs (Figure 2b). QDs PL exhibits a red shift when QDs in solution are deposited on glass forming thin films. In agreement with the absorption red shift (Figure $2 b$ ), after ligand exchange, PbS/FAPI QDs PL presents a red shift with respect to PbS/OA QDs for both solution and thin film. To shed light the PL red shift $\left(\Delta \lambda_{\text {(solution) }}=132 \mathrm{~nm}\right.$ and $\Delta \lambda_{\text {(thin }}$ film $)=211 \mathrm{~nm}$ ) we carry out $\mathrm{k} \cdot \mathrm{p}$ theory calculations of exciton emission. Electron and hole states are computed within a multi-band Dimmock Hamiltonian, including mutual Coulomb interaction via self-consistent process and dielectric mismatch effects, see section 2 in Supporting Information. In Figure 2a we show the experimental PL emission of PbS QDs surrounded by OA or FAPI, either in solution or in film, and compare with theoretical estimates. 

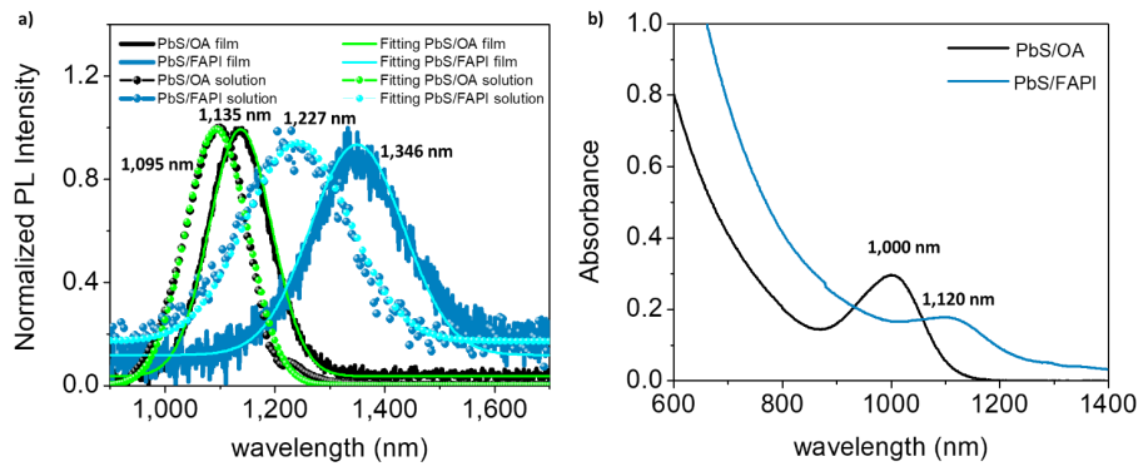

Figure 2. (a) PL steady state spectra of the PbS QDs, $3.8 \mathrm{~nm}$ diameter, in solution (scatter plots) and in thin film (line) with OA (black) and FAPI (blue) as ligands: overlapped to the experimental spectra is the fitting from theoretical calculation with the k.p model (green and cyan lines). (b) IR Absorbance of PbS/OA and PbS/FAPI QDs in solution (octane and DMF respectively). The position of the peak maximum is indicated for both PL and absorption.

Excellent agreement is obtained when the $\mathrm{k} \cdot \mathrm{p}$ model adopts QD diameters of $3.8 \mathrm{~nm}(\mathrm{OA}$, solution), $3.96 \mathrm{~nm}$ (FAPI, solution), $3.98 \mathrm{~nm}$ (OA, film) and $4.42 \mathrm{~nm}$ (FAPI, film), with Gaussianshaped bands to account for size inhomogeneity. The mean size of emitting QDs surrounded by FAPI is slightly larger than that in OA, which is consistent with TEM measurements. Also, the size in films is larger than that in solution, possibly because enhanced tunneling facilitates photoexcited carrier relaxation into larger dots prior to electron-hole recombination. Our theoretical $k \cdot p$ analysis further provides complete information on exciton binding energies, dielectric mismatch effects, band alignment between FAPI and PbS QDs and size-dependent absorption in PbS/FAPI QDs, see Supporting Information section 3 and Figure S2-S6.

The properties of FAPI thin films with embedded PbS QDs have been analyzed as function of the QDs concentration and size. The QDs concentration in the perovskite solution is tuned from $0 \mathrm{mg} / \mathrm{ml}$ (reference sample) to $7.5 \mathrm{mg} / \mathrm{ml}$, resulting in FAPI thin films with different volume percentage of QDs from 0.18 vol \% to 0.55 vol \% (see Supporting Information section 4 for more details about the calculation of the QD percentage). The first beneficial effect of the PbS addition is observed for the crystallization of FAPI film from solution after spin coating. As mentioned above, to produce the black phase of FAPI perovskite an annealing after spin coating at 165-185 
${ }^{\circ} \mathrm{C}$ is needed, but the addition of $\mathrm{PbS}$ reduces dramatically the annealing temperature to obtain the black phase of FAPI, to values as low as $85^{\circ} \mathrm{C}$ for a concentration of PbS/FAPI QDs in solution of $5 \mathrm{mg} / \mathrm{ml}$, see Supporting Video 1, and annealing times as short as 10 minutes. The presence of $\mathrm{PbS}$ accelerates ${ }^{45}$ the FAPI black phase crystallization, not just for FAPI but for reported halide perovskites, reducing radically both the self-assembly time and temperature. ${ }^{16-18}$

The annealing time for the crystallization of the FAPI black phase presents a dependence on the QDs concentration. An optimal response is obtained at $5 \mathrm{mg} / \mathrm{ml}$, with complete conversion into black phase in $10 \mathrm{~min}$. If the concentration is lower or higher, for instance $2.5 \mathrm{mg} / \mathrm{ml}$ or 7.5 $\mathrm{mg} / \mathrm{ml}$, the formation is slowed down to $20 \mathrm{~min}$ and $40 \mathrm{~min}$, respectively, but it still occurs at $85^{\circ} \mathrm{C}$, see Figure S7a. We observe this phenomenon not only with pure DMSO but also with a cosolvent DMF:DMSO (9:1). For the mixture of solvents, the required annealing temperature can be decreased only to $140{ }^{\circ} \mathrm{C}$ (Figure S8a), and the films have a lot of pin-holes on the surface as revealed by Scanning Electron Microscopy (SEM), Figure S8b, compared to the uniform and smooth surfaces of the films obtained from the pure DMSO solution (Figures $3 c-f$ ). This observation is in agreement with previous works pointing out that DMSO accelerates the grain growth of the perovskite, leading to bigger grains, passing from the intermediate adduct with the precursors. ${ }^{46-48}$ However, in this study there is a third component, namely PbS QDs, having a prominent role, as the FAPI black phase crystallizes in DMSO and DMF:DMSO (9:1) at $165^{\circ} \mathrm{C}$ or $185^{\circ} \mathrm{C}$, respectively, without the presence of $\mathrm{PbS}$, see Supporting Video 2, with annealing time longer than 30 minutes to completely convert the precursors in the final product. Summing up, a proper concentration of $\mathrm{PbS}$ QDs results in an impressive shift $\left(\Delta \mathrm{T}=45^{\circ} \mathrm{C}\right.$ if the solvent is $\mathrm{DMF}$ and $\Delta \mathrm{T}=80^{\circ} \mathrm{C}$ if the solvent is the DMSO) of the perovskite starting formation temperature, while reducing the conversion time of the precursors into the black phase. Even in the most recent results on the stabilization of FAPI perovskite black phase with no blue shift of the bandgap, ${ }^{28,34}$ annealing temperatures of $150^{\circ}$ and annealing times longer than 10 minutes are required. This result has the potential in comparison with other approaches to severely reduce production expenses in an eventual industrialization stage.

We have verified that below $85^{\circ} \mathrm{C}$ (i.e. $80^{\circ} \mathrm{C}$ ) an incomplete perovskite formation occurs, as the signatures of $\mathrm{Pbl}_{2}$ and of the FAPI yellow phase are observed in the XRD spectra, see Figure 
S9. On the contrary, the XRD measurements confirm the complete conversion of the perovskite precursors, $\mathrm{FAl}$ and $\mathrm{Pbl}_{2}$, to black FAPI perovskite upon annealing at $85^{\circ} \mathrm{C}$, independently of the $\mathrm{PbS}$ concentration, see Figure 3a. Only the peaks corresponding to the FTO substrate and the FAPI black $\alpha$-phase at $14.03^{\circ}(001), 24.33^{\circ}(111), 28.23^{\circ}(002), 31.66^{\circ}(012)$ and $40.25^{\circ}(022)$ are observed after thin film preparation, see Figure $3 a .{ }^{49}$ No peaks pointing the presence of $\mathrm{Pbl}_{2}$ or FAPI $\delta$-phase are observed after samples preparation. The degradation of these layers was followed at ambient condition, in terms of atmosphere and laboratory illumination, with a relative humidity of almost $45 \%$, without encapsulations. After 30 days, the degradation of the reference sample is evident with bare eyes, see Figure $3 b$, and from absorbance measurements, see Figure S10. For some of the samples with embedded QDs it can be also observed, but not for others. In details, the XRD measurement after 30 days revealed that the FAPI film prepared with a concentration of $\mathrm{PbS}$ QDs of $5 \mathrm{mg} / \mathrm{ml}$ is stable and the black phase is preserved.

a)
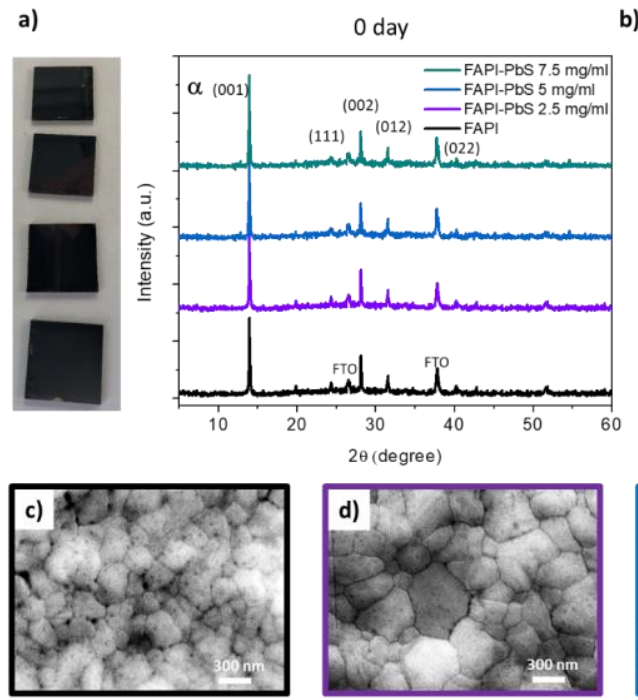

b)
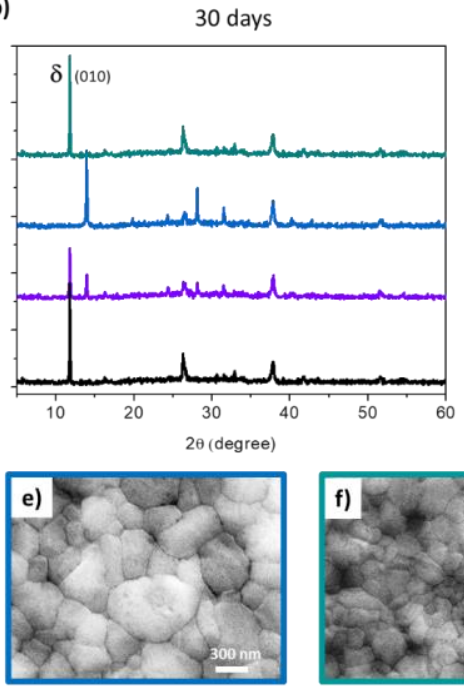

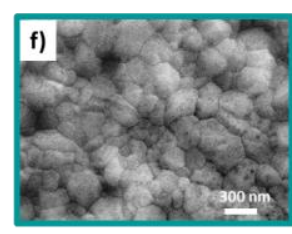

Figure 3. Aging test performed on the perovskite thin films without and with $3.8 \mathrm{~nm}$ size PbS QDs at different concentrations; X-Ray diffraction patterns of (a) the fresh samples and (b) after 30 days, stored in ambient conditions; top-view SEM images of the FAPI thin films (c) without PbS QDs and with PbS QDs (d) $2.5 \mathrm{mg} / \mathrm{ml}$, (e) 5 $\mathrm{mg} / \mathrm{ml}$ and (f) $7.5 \mathrm{mg} / \mathrm{ml}$. The annealing temperature and time for the samples without PbS QDs are $180{ }^{\circ} \mathrm{C}$ for 30 $\mathrm{min}$, and for the samples with the three different PbS QDs concentrations are $85^{\circ} \mathrm{C}$ for $10 \mathrm{~min}$. 
On the other hand, the perovskite without PbS QDs and with the highest concentration embedded, are totally converted into the $\delta$-phase, as indicated by the diffraction peak at $11.8^{\circ}$ and the disappearance of black phase peaks, see Figure $3 \mathrm{~b}$. In the case of the sample with the lowest QD concentration, it presents a mixture of the FAPI black and yellow phases, see Figure 3b. Likewise, the FAPI perovskite grain size increases with the increase of the concentration, but at $7.5 \mathrm{mg} / \mathrm{ml}$ the grain size decreases again, as the SEM images display, see Figure $3 \mathrm{c}-\mathrm{f}$. The black dots observed in the top-view SEM image of the FAPI with no QDs and with PbS QDs $7.5 \mathrm{mg} / \mathrm{ml}$ are yet another evidence of the fast degradation of such films when exposed to ambient air or to stress conditions, like the SEM beam. No such signs are observed for the sample with $5 \mathrm{mg} / \mathrm{ml}$ QDs.

The size of the PbS QDs also has a significant effect on the stabilization of FAPI black phase. To illustrate this point, we keep the concentration in solution constant, at $5 \mathrm{mg} / \mathrm{ml}$, and vary the PbS QDs size. PbS QDs with diameter size of $2.9 \mathrm{~nm}, 3.8 \mathrm{~nm}$ and $8.2 \mathrm{~nm}$ are studied. PL, TEM images and XRD of the corresponding PbS QDs are plotted in Figure S11. An optimal response is obtained for the mid-sized QD diameter, $3.8 \mathrm{~nm}$. The trends of annealing time and black phase stability when varying the size from this value are analogous to those observed when varying the concentration from its optimal value, see Figure S7. Namely, the annealing time is shortened from the system with PbS QDs of $2.9 \mathrm{~nm}$ to the system with $3.8 \mathrm{~nm}$ and increases again for PbS QDs of $8.2 \mathrm{~nm}$. In the same way, the stability improves from $2.9 \mathrm{~nm}$ to $3.8 \mathrm{~nm}$, but it decreases for the largest size.

One of the most significant advantages of the FA-based perovskite compared with the MA-based perovskite, in addition to the lower band gap, is the thermal stability beyond $150{ }^{\circ} \mathrm{C}$, as it can be expected from the higher formation energy. Despite the introduction of PbS QDs reduces significantly the annealing temperature for the synthesis of FAPI black phase, the presence of QDs does not affect the thermal stability of the FAPI layer, as it has been verified by thermogravimetric analysis (TGA). TGA was measured from room temperature to $1,000{ }^{\circ} \mathrm{C}$ under nitrogen, Figure S12, observing no significant differences for samples prepared with and without QDs. 
In order to understand the physical origin of the phase stability of FAPI in the presence of embedded PbS QDs, density functional theory (DFT) calculations including spin-orbit coupling are carried out (see Supporting Information, section 5, Figure S13-S16). We compare the energetic stability of black and yellow phases in different conditions. As shown in panel (i) of Figure 4a, in the bulk the energy of black (cubic) phase exceeds that of the yellow one by $\Delta E_{b y}=E_{b}-E_{y}=0.27$ eV per $\mathrm{FAPbl}_{3}$ unit. This fact explains the well-known thermodynamic preference for the yellow phase in the absence of PbS QDs. ${ }^{9}$ Upon inclusion of the QDs, however, several changes take place in the host FAPI matrix: (i) the smaller lattice constant of PbS generates strain, (ii) crystal surfaces arise on the interface with the QD and (iii) chemical bonds form between the two materials. We study the individual effect of each factor to determine their presumable role in stabilizing the black phase.

First, we investigate the effect of strain. Previous studies have suggested strain may favor the black phase stability in $\mathrm{CsPb}_{3}$ and $(\mathrm{Cs}, \mathrm{FA}) \mathrm{Pbl}_{3}$ perovskites with different substrates. ${ }^{50-52} \mathrm{We}$ calculate the strain arising from the lattice mismatch between spherical PbS QDs and cubic FAPI using linear elastic theory (see details in section 2 of Supporting Information). Figure 4b shows the strain parallel ( $\|$ ) and normal $(\perp)$ to the (100) crystal direction for QDs of different sizes. We find most of the strain occurs in the FAPI matrix, as it stays well under $1 \%$ inside the QDs, but it becomes strong (up to 4-6 \%) outside. This is consistent with the rigid (soft) bonds of PbS (FAPI). A strongly compressive strain $\varepsilon_{\perp}$ builds up in FAPI near the interface with PbS (dashed lines), which facilitates lattice matching. In response, a tensile strain $\varepsilon \|$ (solid lines) develops in the orthogonal direction, which propagates several nanometers away from the interface. This behavior implies a long-ranged tetragonal distortion of the FAPI lattice, as sketched in Figure 4c. The energetic impact of such a strain is determined by DFT calculations. The isolated effect of the strain on the bulk is evaluated by forcing that the bulk perovskite matches with the PbS lattice: black (cubic) and yellow (hexagonal) bulk phases are forced to match the lattice constant of PbS on (100) and (111) surfaces, respectively, which are the most compatible crystallographic planes in each case. The in-plane (out-of-plane) lattice parameters are compressed (tensiled) by $7 \%$ on axis $a$ and $b$ ( $12 \%$ on $c$ axis) for the black phase -which becomes tetragonal- and by $13 \%$ on $a$ axis and $2 \%$ on $b$ axis ( $8 \%$ on $c$ axis) for the yellow phase. The resulting energies show that strain 
destabilizes both phases, but the effect is more pronounced in the yellow one. Thus, the energy splitting decreases from $\Delta \mathrm{E}_{\mathrm{by}}=0.27 \mathrm{eV}$ to $\Delta \mathrm{E}_{\mathrm{by}}=0.18 \mathrm{eV}$, as shown in Figure 4a, cf. panels (i) and (ii). A similar energetic trend has been recently reported in Ref. ${ }^{50}$ for $\mathrm{CsPbl}_{3}$ thin films depending on the strain induced by the substrate. However, the small reduction is unlikely to explain the drastic stabilization of the black phase revealed in our experiments. We have tested biaxial and uniaxial strain on bulk crystals, both tensile and compressive, and confirmed that the black phase is systematically less stable than the yellow one, i.e. $\Delta \mathrm{E}_{\mathrm{by}}>0$, see Figure S17.

We next study the effect of surface formation. PbS QDs of small size present an octahedral shape exposing (111) planes, while QDs with diameters over $\sim 3.5 \mathrm{~nm}$ PbS QDs adopt a cubooctahedral shape with increasing area covered by (100) planes, see Figure 4d. ${ }^{53}$ FAPI black phase is prone to use (100) planes to bind with PbS, while the yellow phase prefers (111) planes, each with a preferred ionic termination. A quantitative comparison of the corresponding surface energies is however prevented by the lack of reflection symmetry in the unit cell of the yellow phase, its inherent polarity, and the wide uncertainty range of FAPI chemical potential. We then carry out a semi-quantitative analysis of surface energies by directly comparing the total electronic energy of strained slabs in black and yellow phases. The slabs, as plotted in panel (iii) of Figure $4 a$, are chosen to have similar thicknesses and hence similar quantum confinement. As expected, the presence of surfaces increases the energy per $\mathrm{FAPbl}_{3}$ unit with respect to strained bulk. The increase is small for the black phase but large for the yellow one, which then becomes less stable than the black phase by $\Delta \mathrm{E}_{\mathrm{by}}=-0.76 \mathrm{eV}$. This result is consistent with recent studies on $\mathrm{CsPb}_{3}$, where large (small) surface energies were reported for the $\delta(\alpha)$ phase. ${ }^{52}$ It follows that the presence of surfaces between FAPI and PbS QDs is an important factor destabilizing the yellow phase relative to the black one. ${ }^{50}$

The last factor to be considered is the chemical binding of FAPI to PbS. For a semiquantitative estimate, we attach slabs of FAPI and PbS, as illustrated in panel (iv) of Figure 4a. The resulting energy per $\mathrm{FAPbl}_{3}$ unit decreases for both phases as compared to that of bare slabs, which confirms that stable bonds are formed between the two materials. Remarkably, the stabilization is much stronger for the black phase than for the yellow one, the energy difference increasing from $\Delta \mathrm{E}_{\mathrm{by}}=-0.76 \mathrm{eV}$ in the free-standing slabs to $\Delta \mathrm{E}_{\mathrm{by}}=-1.06 \mathrm{eV}$ in the heterostructure. 
Altogether, the energetic trends revealed by our DFT calculations (see SI for more details), make clear that the origin of thermodynamically favored black FAPI upon inclusion of PbS QDs is due mainly to two distinct, but both needed, mechanisms: in primis the structure stabilization, that destabilizes the yellow phase due to its large surface energy and in secundis the crucial chemical stabilization, by chemical bonds between the PbS and FAPI, that stabilizes the black one phase. The latter is also likely related to the mild annealing conditions observed in our experiments. The low annealing temperature indicates $\mathrm{PbS}$ QDs are acting as catalyzers, ${ }^{45}$ regardless of the size and concentration, which reduce the activation energy required to attain the metastable black phase. It is then plausible that the strong PbS-black FAPI bonds are stabilizing the transition state of the reaction path. Strain, on the other hand, plays a secondary role in energetic stabilization. Still, the long-ranged tetrahedral distortion reported in Figure $4 \mathrm{~b}$ suggests PbS QDs could further contribute to stabilize by stimulating the formation of large FAPI grains black phase FAPI. It has been recently claimed that the strain enables the formation of large grain sizes through the coherent propagation of its field over crystal regions with otherwise random orientation of domains, ${ }^{50}$ which is beneficial for the stability of the synthesized phase. ${ }^{9}$

The results above clearly explain the experimental observation that small size PbS QDs and small concentrations of QDs fail to stabilize the FAPI black phase, because of the lack of (100) surfaces and the reduced number of surfaces and chemical bonds, respectively. A possible explanation to understand the lower stability for high QDs concentration and higher QD size, is that the presence of QDs rich regions can produce a large increase of the strain, see Figure S18a,b, especially in the case of large QDs size. This significant strain enhancement produces in turn an important increase of the energy per formula unit, see Figure S17, which could induce the observed destabilization of the thin film. In order to experimentally prove the effect of the strain, the shift of the XRD peaks is also analyzed. FAPI with and without QDs powders have been prepared in order to ensure enough quantity of material for characterization and remove the effect of the substrate. Powder were obtained by precipitation method, see Supporting Information for further details. A shift in the XRD diffraction peak, increasing with the amount of QD concentration is observed, see Figure S18c-d. Fitting the data with Williamson-Hall method ${ }^{54,55}$ we found that as expected the strain increases with the amount of QDs, due to a more compressed lattice, see Figure S18e. 
Both QDs size and proximity between QDs enhance strain fields, see Figure S18a-b, as theoretically and experimentally demonstrated. A possible explanation for the lower stability of samples with a high concentration of QDs is that the energetic destabilization near to the QDs makes distant regions detach and form independent grains with no strain, more stable in the yellow phase. Another possibility is that large QDs have larger percentage of (100) facets, and when this percentage is too large QD can start to attach to nearby dots, ${ }^{56}$ producing agglomeration and they may become less useful for FAPI stabilization. Moreover, more macroscopic effect also could play a role, as higher concentrations result in a higher density of nucleation sites, which should also lead to smaller grain sizes, increasing the amount of grain boundaries, known to be detrimental for the black phase stability e.g. by enabling humidity filtration. ${ }^{19}$

a)

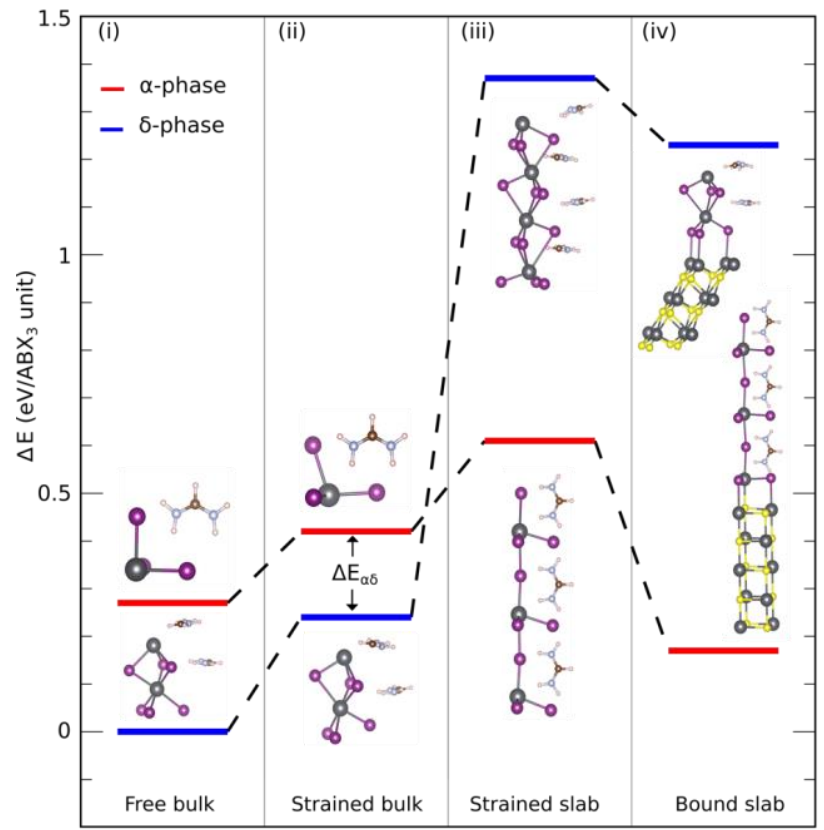

b)

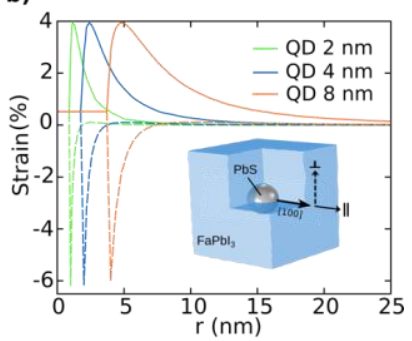

c) Unstrained

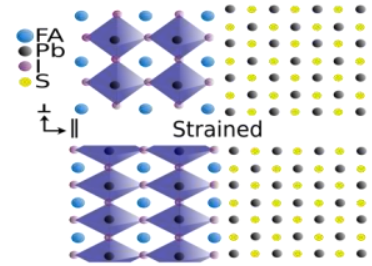

d)

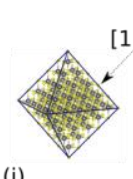

(ii)

Figure 4. (a) Total energy DFT calculation of (i) black and yellow bulk FAPI phases represented by red and blue colors respectively, (ii) the effect of strain on the bulk when matching the PbS lattice, (iii) the presence of surfaces in the slabs, and (iv) the role of chemical binding to the PbS substrate in the heterojunctions. The insets depict the atomic structures under study, which are 3D in (i) and (ii), and 2D in (iii) and (iv). (b) Strain tensor elements parallel and perpendicular to (100) axis, for three different QD diameters. The (100) direction is normal to the FAPI-PbS interface. (c) Sketch of the effect of strain in the lattice, as inferred from b). A tetragonal distortion is observed, which 
penetrates several $\mathrm{nm}$ inside FAPI. (d) Atomic representation of PbS quantum dots showing facets (111) and (100).

The small dot in panel (i) is $3 \mathrm{~nm}$ size and the larger one in panel (ii) is $4.2 \mathrm{~nm}$ size.

To investigate the effects of the PbS QDs incorporation in photovoltaic devices, we

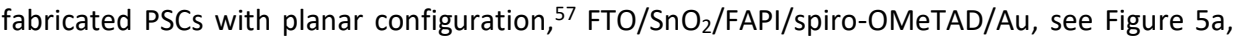
with and without embedded QDs. We employed the optimized PbS QDs solution concentration of $5 \mathrm{mg} / \mathrm{ml}$ and QD size of $3.8 \mathrm{~nm}$. The average of the results is summarized in Figure S19a-d and Table S3 and the curves of the best devices are shown in Figure 5b. We highlight that the presence of PbS QDs not only increases the maximum and average performance of the solar cells but also the reproducibility of the devices, see Figure S19d, due to the augmentation of the open-circuit voltage, $\mathrm{V}_{\mathrm{oc}}$, and the fill factor, FF, see Figure S19b and S19c, respectively, for average values and Figure $5 b$ for champion devices. Significantly, the champion device has a stable photo-conversion efficiency of $18 \%$, see Table S3, Figure $5 \mathrm{~b}$ and Figure S20a, with a $\mathrm{V}_{\text {oc }}$ of $1,105 \mathrm{mV}$, a photocurrent, $\mathrm{J}_{\mathrm{sc}}$ of $21.5 \mathrm{~mA} / \mathrm{cm}^{2}$ and a FF of $75.7 \%$, with typical negligible hysteresis, in accordance with previous reports. ${ }^{58}$ However, the solar cells optimization is not exhausted, as the theoretical limit of $27 \mathrm{~mA} / \mathrm{cm}^{2}$ suggests, ${ }^{58}$ and further optimization can be expected in the near future. An increase of the layer thickness (higher than $220 \mathrm{~nm}$ ) optimizing the deposition process, keeping the FF as high as in this study, will allow to take benefit of the lower band gap of FAPI, see Figure $5 e$, to increase the device $J_{s c}$ (Figure $S 19$ e-i). 
a)

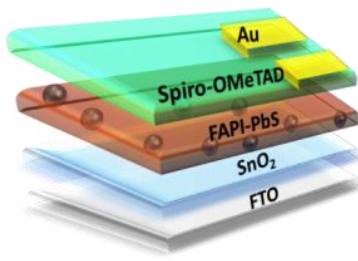

b) 25

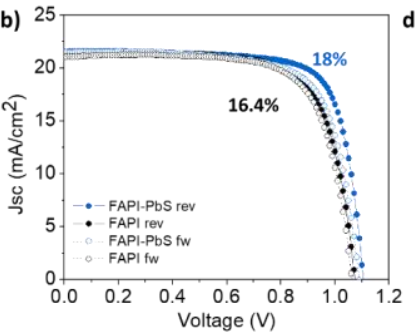

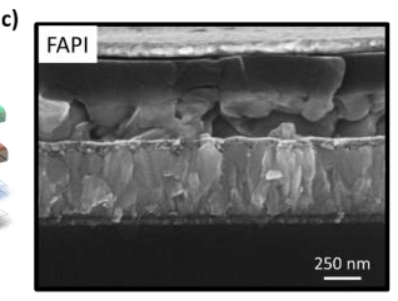

d)

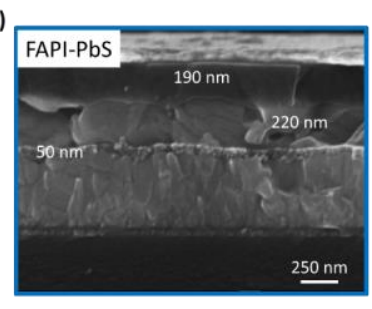

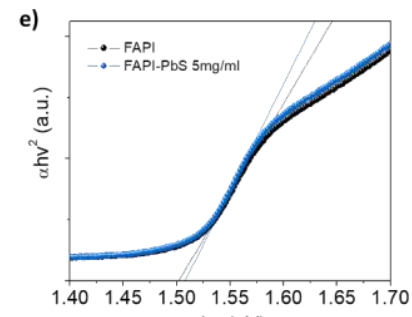

hv (eV)

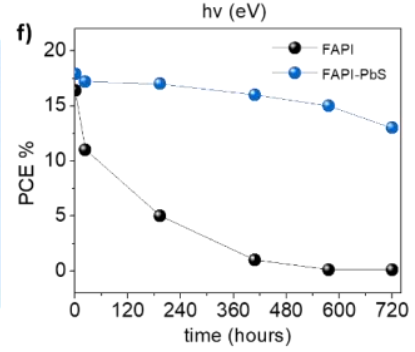

Figure 5| Photovoltaic device J/V characterization. a, Sketch of the architecture used in this study; b, Current/Voltage curves of the best performance perovskite solar cells recorded at reverse and forward scan; c, and d, SEM cross sections of the devices with the two different active layers FAPI and FAPI-PbS; e, Tauc plot of the FAPI and FAPI with $5 \mathrm{mg} / \mathrm{ml}$; the estimated band gap is $1.50 \mathrm{eV}$; $\mathbf{f}$, Stability of the perovskite solar cells during the days.

Figure S20 shows the incident photon-to-current efficiency (IPCE \%) and the integrated current $\mathrm{J}_{\mathrm{sc}}$, corresponding to the value collected with the J/V curves measurements. The value of the integrated current of the control device, because of the fast degradation of the film, is lower than the initial value, while there is a good agreement with the measured $J_{s c}$ in the case of samples with QDs. On the other hand, the $\mathrm{SnO}_{2} / \mathrm{FAPI}-\mathrm{PbS}$ interface is more compact if compared to the layer without PbS QDs, see Figure 5c-d, thus confirming that the PbS QDs also positively affect the morphology enhancing the device performance, in particular the $\mathrm{V}_{o c}$. The obtained $\mathrm{V}_{o c}$ in fact increases reaching a promising value of $1,105 \mathrm{mV}, 91 \%$ of the maximum theoretical $V_{o c}$ of $1,215 \mathrm{mV}$ for pure FAPI perovskite with a band gap of $1.5 \mathrm{eV},{ }^{8}$ see Figure $5 \mathrm{e}$. To our knowledge, this is the highest $V_{o c}$ reported for pure FAPI PSCs. Beyond the improvement of the PCE, PbS QDs provide a drastic enhancement of long term stability, see Figure $5 f$. Devices prepared with QDs preserve $85 \%$ of their performance after 720 hours, while samples without QDs decrease their 
performance by $30 \%$ after a single day since fabrication, and show no performance at all after 500 hours.

In conclusion, we have shown that pure FAPI perovskite, preserving its excellent optical properties for solar cell devices, can be stabilized by embedding a small fraction of PbS QDs. As compared to previous strategies, mostly based on ion alloying, the present method avoids blue shifting of the band gap -thus paving the way for higher PCE- and reduces dramatically both synthesis temperature and time -thus reducing production expenses -. The stabilization is based on the double contribution of the PbS-FAPI interface, chemical and structural, which selectively favors black phase against yellow one. This work opens venue for crystalline phase stabilization of metastable polymorphs through surface chemistry engineering, not just in very thin films under the effect of a substrate, but in bulky systems by the intentional creation of surfaces and chemical bonds with embedded QDs.

\section{- ASSOCIATED CONTENT}

\section{Supporting Information}

The Supporting Information is available free of charge on the ACS Publications website

Experimental section, Theoretical $k \cdot p$ model and materials parameters, Additional Theoretical Calculations, Percentage of volume occupied by QDs, Density Functional Theory Calculations, Figures S1-S18.

\section{- AUTHOR INFORMATION}

\section{Corresponding Authors}

*E-mail: masi@uji.es (S.M.); climente@uji.es (J.I.C.); sero@uji.es (I.M.-S.)

Twitter: @sofy_masi (S.M.); @IvanMoraSero (I.M.-S.)

Notes

The authors declare no competing financial interest.

\section{- ACKNOWLEDGEMENTS}

Financial support from the European Research Council (ERC) via Consolidator Grant (724424-No-LIMIT) and Generalitat Valenciana via Prometeo Grant Q-Devices (Prometeo/2018/098) is gratefully 
acknowledged. P.F Méndez thank Fundación Carolina and Universidad Autónoma de Sinaloa for short stay fellowship. The work of E. López-Fraguas was supported by the Ministerio de Educación y Formación Profesional through his FPU Research Fellowship under Grant FPU17/00612. JP and JIC acknowledge support from UJI B2017-59 project. Servei Central d'Instrumentació Científica (SCIC) from Universitat Jaume $I$ is acknowledged for its help with SEM, XRD and FTIR measurements.

\section{- AUTHOR CONTRIBUTIONS}

S.M. and I.M.-S. conceived the project. S.M. and S.K.P.M. optimized the ligand exchange and T.T.N synthetized and characterized by PL of the PbS QDs. S.M prepared the solar cells and characterized them. P.M. prepared the samples and performed the SEM. E. F. L performed the absorbance and Tauc plot. C.E.A developed the DFT simulations and J.I.C., J.P. and D.F.M.P. the strain theoretical calculations and the k.p model analysis. S.M. coordinated the experimental work. J.I.C. coordinated the theoretical work. I.M.-S. coordinated the whole project. S.M, J.I.C. and I.M.-S. wrote the manuscript. All the authors contributed to the discussions.

\section{References}

(1) https://www.nrel.gov/pv/assets/pdfs/best-research-cell-efficiencies.20190802.pdf.

(2) Fakharuddin, A.; Shabbir, U.; Qiu, W.; Iqbal, T.; Sultan, M.; Heremans, P.; Schmidt-Mende, L. Inorganic and Layered Perovskites for Optoelectronic Devices. Advanced Materials 2019, 31, 1807095.

(3) Gholipour, S.; Ali, A. M.; Correa-Baena, J.-P.; Turren-Cruz, S.-H.; Tajabadi, F.; Tress, W.; Taghavinia, N.; Grätzel, M.; Abate, A.; De Angelis, F.; Gaggioli, C. A.; Mosconi, E.; Hagfeldt, A.; Saliba, M. Globularity-Selected Large Molecules for a New Generation of Multication Perovskites. Advanced Materials 2017, 29, 1702005.

(4) Shockley, W.; Queisser, H. J. Detailed Balance Limit of Efficiency of p-n Junction Solar Cells. Journal of Applied Physics 1961, 32, 510-519.

(5) Amat, A.; Mosconi, E.; Ronca, E.; Quarti, C.; Umari, P.; Nazeeruddin, M. K.; Grätzel, M.; De Angelis, F. Cation-Induced Band-Gap Tuning in Organohalide Perovskites: Interplay of Spin-Orbit Coupling and Octahedra Tilting. Nano Letters 2014, 14, 3608-3616.

(6) Yang, W. S.; Noh, J. H.; Jeon, N. J.; Kim, Y. C.; Ryu, S.; Seo, J.; Seok, S. I. High-performance photovoltaic perovskite layers fabricated through intramolecular exchange. Science 2015, 348, 1234-1237.

(7) Koh, T. M.; Fu, K.; Fang, Y.; Chen, S.; Sum, T. C.; Mathews, N.; Mhaisalkar, S. G.; Boix, P. P.; Baikie, T. Formamidinium-Containing Metal-Halide: An Alternative Material for Near-IR Absorption Perovskite Solar Cells. The Journal of Physical Chemistry C 2014, 118, 16458-16462.

(8) Rühle, S. Tabulated values of the Shockley-Queisser limit for single junction solar cells. Solar Energy 2016, 130, 139-147.

(9) Zhou, Y.; Zhao, Y. Chemical stability and instability of inorganic halide perovskites. Energy \& Environmental Science 2019, 12, 1495-1511. 
(10) Li, Z.; Yang, M.; Park, J.-S.; Wei, S.-H.; Berry, J. J.; Zhu, K. Stabilizing Perovskite Structures by Tuning Tolerance Factor: Formation of Formamidinium and Cesium Lead lodide Solid-State Alloys. Chemistry of Materials 2016, 28, 284-292.

(11) Yi, C.; Luo, J.; Meloni, S.; Boziki, A.; Ashari-Astani, N.; Grätzel, C.; Zakeeruddin, S. M.; Röthlisberger, U.; Grätzel, M. Entropic stabilization of mixed A-cation ABX3 metal halide perovskites for high performance perovskite solar cells. Energy \& Environmental Science 2016, 9, 656-662.

(12) Saliba, M.; Matsui, T.; Seo, J.-Y.; Domanski, K.; Correa-Baena, J.-P.; Nazeeruddin, M. K.; Zakeeruddin, S. M.; Tress, W.; Abate, A.; Hagfeldt, A.; Grätzel, M. Cesium-containing triple cation perovskite solar cells: improved stability, reproducibility and high efficiency. Energy \& Environmental Science 2016, 9, 1989-1997.

(13) Fabini, D. H.; Stoumpos, C. C.; Laurita, G.; Kaltzoglou, A.; Kontos, A. G.; Falaras, P.; Kanatzidis, M. G.; Seshadri, R. Reentrant Structural and Optical Properties and Large Positive Thermal Expansion in Perovskite Formamidinium Lead lodide. Angewandte Chemie International Edition 2016, 55, 15392-15396.

(14) Weber, O. J.; Ghosh, D.; Gaines, S.; Henry, P. F.; Walker, A. B.; Islam, M. S.; Weller, M. T. Phase Behavior and Polymorphism of Formamidinium Lead lodide. Chemistry of Materials 2018, 30, 3768-3778.

(15) Stoumpos, C. C.; Malliakas, C. D.; Kanatzidis, M. G. Semiconducting Tin and Lead lodide Perovskites with Organic Cations: Phase Transitions, High Mobilities, and Near-Infrared Photoluminescent Properties. Inorganic Chemistry 2013, 52, 9019-9038.

(16) Zhang, Y.; Kim, S.-G.; Lee, D.-K.; Park, N.-G. CH3NH3Pbl3 and HC(NH2)2Pbl3 Powders Synthesized from Low-Grade Pbl2: Single Precursor for High-Efficiency Perovskite Solar Cells. ChemSusChem 2018, 11, 1813-1823.

(17) Pool, V. L.; Dou, B.; Van Campen, D. G.; Klein-Stockert, T. R.; Barnes, F. S.; Shaheen, S. E.; Ahmad, M. I.; van Hest, M. F. A. M.; Toney, M. F. Thermal engineering of FAPbI3 perovskite material via radiative thermal annealing and in situ XRD. Nature Communications 2017, 8, 14075.

(18) Dualeh, A.; Tétreault, N.; Moehl, T.; Gao, P.; Nazeeruddin, M. K.; Grätzel, M. Effect of Annealing Temperature on Film Morphology of Organic-Inorganic Hybrid Pervoskite Solid-State Solar Cells. Advanced Functional Materials 2014, 24, 3250-3258.

(19) Cordero, F.; Craciun, F.; Trequattrini, F.; Generosi, A.; Paci, B.; Paoletti, A. M.; Pennesi, G. Stability of Cubic FAPbI3 from X-ray Diffraction, Anelastic, and Dielectric Measurements. The Journal of Physical Chemistry Letters 2019, 10, 2463-2469.

(20) Jiang, Q.; Zhao, Y.; Zhang, X.; Yang, X.; Chen, Y.; Chu, Z.; Ye, Q.; Li, X.; Yin, Z.; You, J. Surface passivation of perovskite film for efficient solar cells. Nature Photonics 2019, 13, 460-466.

(21) McMeekin, D. P.; Sadoughi, G.; Rehman, W.; Eperon, G. E.; Saliba, M.; Hörantner, M. T.; Haghighirad, A.; Sakai, N.; Korte, L.; Rech, B.; Johnston, M. B.; Herz, L. M.; Snaith, H. J. A mixedcation lead mixed-halide perovskite absorber for tandem solar cells. Science 2016, 351, 151-155.

(22) Que, M.; Dai, Z.; Yang, H.; Zhu, H.; Zong, Y.; Que, W.; Padture, N. P.; Zhou, Y.; Chen, O. QuantumDot-Induced Cesium-Rich Surface Imparts Enhanced Stability to Formamidinium Lead lodide Perovskite Solar Cells. ACS Energy Letters 2019, 4, 1970-1975.

(23) Lee, J.-W.; Kim, D.-H.; Kim, H.-S.; Seo, S.-W.; Cho, S. M.; Park, N.-G. Formamidinium and Cesium Hybridization for Photo- and Moisture-Stable Perovskite Solar Cell. Advanced Energy Materials 2015, 5, 1501310.

(24) Yao, D.; Zhang, C.; Pham, N. D.; Zhang, Y.; Tiong, V. T.; Du, A.; Shen, Q.; Wilson, G. J.; Wang, H. Hindered Formation of Photoinactive $\delta$-FAPbl3 Phase and Hysteresis-Free Mixed-Cation Planar Heterojunction Perovskite Solar Cells with Enhanced Efficiency via Potassium Incorporation. The Journal of Physical Chemistry Letters 2018, 9, 2113-2120. 
(25) Saliba, M.; Matsui, T.; Domanski, K.; Seo, J.-Y.; Ummadisingu, A.; Zakeeruddin, S. M.; CorreaBaena, J.-P.; Tress, W. R.; Abate, A.; Hagfeldt, A.; Grätzel, M. Incorporation of rubidium cations into perovskite solar cells improves photovoltaic performance. Science 2016, 354, 206-209.

(26) Lee, J.-W.; Dai, Z.; Han, T.-H.; Choi, C.; Chang, S.-Y.; Lee, S.-J.; De Marco, N.; Zhao, H.; Sun, P.; Huang, Y.; Yang, Y. 2D perovskite stabilized phase-pure formamidinium perovskite solar cells. Nature Communications 2018, 9, 3021.

(27) Niu, T.; Lu, J.; Tang, M.-C.; Barrit, D.; Smilgies, D.-M.; Yang, Z.; Li, J.; Fan, Y.; Luo, T.; McCulloch, I.; Amassian, A.; Liu, S.; Zhao, K. High performance ambient-air-stable FAPbl3 perovskite solar cells with molecule-passivated Ruddlesden-Popper/3D heterostructured film. Energy \& Environmental Science 2018, 11, 3358-3366.

(28) Alanazi, A. Q.; Kubicki, D. J.; Prochowicz, D.; Alharbi, E. A.; Bouduban, M. E. F.; Jahanbakhshi, F.; Mladenovic, M.; Milic, J. V.; Giordano, F.; Ren, D.; Alyamani, A. Y.; Albrithen, H.; Albadri, A.; Alotaibi, M. H.; Moser, J.-E.; Zakeeruddin, S. M.; Rothlisberger, U.; Emsley, L.; Grätzel, M. AtomicLevel Microstructure of Efficient Formamidinium-Based Perovskite Solar Cells Stabilized by 5Ammonium Valeric Acid lodide Revealed by Multi-Nuclear and Two-Dimensional Solid-State NMR. Journal of the American Chemical Society 2019, 141, 17659-17669.

(29) Eperon, G. E.; Stranks, S. D.; Menelaou, C.; Johnston, M. B.; Herz, L. M.; Snaith, H. J. Formamidinium lead trihalide: a broadly tunable perovskite for efficient planar heterojunction solar cells. Energy \& Environmental Science 2014, 7, 982-988.

(30) Pellet, N.; Gao, P.; Gregori, G.; Yang, T.-Y.; Nazeeruddin, M. K.; Maier, J.; Grätzel, M. MixedOrganic-Cation Perovskite Photovoltaics for Enhanced Solar-Light Harvesting. Angewandte Chemie International Edition 2014, 53, 3151-3157.

(31) Prasanna, R.; Gold-Parker, A.; Leijtens, T.; Conings, B.; Babayigit, A.; Boyen, H.-G.; Toney, M. F.; McGehee, M. D. Band Gap Tuning via Lattice Contraction and Octahedral Tilting in Perovskite Materials for Photovoltaics. Journal of the American Chemical Society 2017, 139, 11117-11124.

(32) Haque, F.; Yi, H.; Duan, L.; Zhang, Y.; Wright, M.; Conibeer, G.; Uddin, A. Optimisation of annealing temperature for low temperature processed inverted structure Caesium Formamidinium Lead Triiodide perovskite solar cells. Materials Science in Semiconductor Processing 2019, 102, 104580.

(33) Turren-Cruz, S.-H.; Hagfeldt, A.; Saliba, M. Methylammonium-free, high-performance, and stable perovskite solar cells on a planar architecture. Science 2018, 362, 449-453.

(34) Min, H.; Kim, M.; Lee, S.-U.; Kim, H.; Kim, G.; Choi, K.; Lee, J. H.; Seok, S. I. Efficient, stable solar cells by using inherent bandgap of $\alpha$-phase formamidinium lead iodide. Science 2019, 366, 749753.

(35) Ngo, T. T.; Mora-Seró, I. Interaction between Colloidal Quantum Dots and Halide Perovskites: Looking for Constructive Synergies. The Journal of Physical Chemistry Letters 2019, 10, 1099-1108.

(36) Ngo, T. T.; Suarez, I.; Sanchez, R. S.; Martinez-Pastor, J. P.; Mora-Sero, I. Single step deposition of an interacting layer of a perovskite matrix with embedded quantum dots. Nanoscale 2016, 8, 14379-14383.

(37) Ning, Z.; Gong, X.; Comin, R.; Walters, G.; Fan, F.; Voznyy, O.; Yassitepe, E.; Buin, A.; Hoogland, S.; Sargent, E. H. Quantum-dot-in-perovskite solids. Nature 2015, 523, 324.

(38) Gong, X.; Yang, Z.; Walters, G.; Comin, R.; Ning, Z.; Beauregard, E.; Adinolfi, V.; Voznyy, O.; Sargent, E. H. Highly efficient quantum dot near-infrared light-emitting diodes. Nature Photonics 2016, 10 , 253.

(39) García de Arquer, F. P.; Gong, X.; Sabatini, R. P.; Liu, M.; Kim, G.-H.; Sutherland, B. R.; Voznyy, O.; $\mathrm{Xu}$, J.; Pang, Y.; Hoogland, S.; Sinton, D.; Sargent, E. Field-emission from quantum-dot-inperovskite solids. Nature Communications 2017, 8, 14757.

(40) Han, J.; Luo, S.; Yin, X.; Zhou, Y.; Nan, H.; Li, J.; Li, X.; Oron, D.; Shen, H.; Lin, H. Hybrid PbS QuantumDot-in-Perovskite for High-Efficiency Perovskite Solar Cell. Small 2018, 14, 1801016. 
(41) Liu, M.; Chen, Y.; Tan, C.-S.; Quintero-Bermudez, R.; Proppe, A. H.; Munir, R.; Tan, H.; Voznyy, O.; Scheffel, B.; Walters, G.; Kam, A. P. T.; Sun, B.; Choi, M.-J.; Hoogland, S.; Amassian, A.; Kelley, S. O.; García de Arquer, F. P.; Sargent, E. H. Lattice anchoring stabilizes solution-processed semiconductors. Nature 2019, 570, 96-101.

(42) Dirin, D. N.; Dreyfuss, S.; Bodnarchuk, M. I.; Nedelcu, G.; Papagiorgis, P.; Itskos, G.; Kovalenko, M. V. Lead Halide Perovskites and Other Metal Halide Complexes As Inorganic Capping Ligands for Colloidal Nanocrystals. Journal of the American Chemical Society 2014, 136, 6550-6553.

(43) Moreels, I.; Lambert, K.; Smeets, D.; De Muynck, D.; Nollet, T.; Martins, J. C.; Vanhaecke, F.; Vantomme, A.; Delerue, C.; Allan, G.; Hens, Z. Size-Dependent Optical Properties of Colloidal PbS Quantum Dots. ACS Nano 2009, 3, 3023-3030.

(44) He, Y.; Yoon, Y. J.; Harn, Y. W.; Biesold-McGee, G. V.; Liang, S.; Lin, C. H.; Tsukruk, V. V.; Thadhani, N.; Kang, Z.; Lin, Z. Unconventional route to dual-shelled organolead halide perovskite nanocrystals with controlled dimensions, surface chemistry, and stabilities. Science Advances 2019, 5, eaax4424.

(45) Lin, C.-Y.; Li, S.-S.; Chang, J.-W.; Chia, H.-C.; Hsiao, Y.-Y.; Su, C.-J.; Lian, B.-J.; Wen, C.-Y.; Huang, S.K.; Wu, W.-R.; Wang, D.-Y.; Su, A.-C.; Chen, C.-W.; Jeng, U.-S. Unveiling the Nanoparticle-Seeded Catalytic Nucleation Kinetics of Perovskite Solar Cells by Time-Resolved GIXS. Advanced Functional Materials 2019, 29, 1902582.

(46) Li, W.; Fan, J.; Li, J.; Mai, Y.; Wang, L. Controllable Grain Morphology of Perovskite Absorber Film by Molecular Self-Assembly toward Efficient Solar Cell Exceeding 17\%. Journal of the American Chemical Society 2015, 137, 10399-10405.

(47) Liu, T.; Zong, Y.; Zhou, Y.; Yang, M.; Li, Z.; Game, O. S.; Zhu, K.; Zhu, R.; Gong, Q.; Padture, N. P. High-Performance Formamidinium-Based Perovskite Solar Cells via Microstructure-Mediated $\delta$ to- $\alpha$ Phase Transformation. Chemistry of Materials 2017, 29, 3246-3250.

(48) Meng, L.; Sun, C.; Wang, R.; Huang, W.; Zhao, Z.; Sun, P.; Huang, T.; Xue, J.; Lee, J.-W.; Zhu, C.; Huang, Y.; Li, Y.; Yang, Y. Tailored Phase Conversion under Conjugated Polymer Enables Thermally Stable Perovskite Solar Cells with Efficiency Exceeding 21\%. Journal of the American Chemical Society 2018, 140, 17255-17262.

(49) Weller, M. T.; Weber, O. J.; Frost, J. M.; Walsh, A. Cubic Perovskite Structure of Black Formamidinium Lead lodide, $\alpha-\left[\mathrm{HC}\left(\mathrm{NH}_{2}\right) 2\right] \mathrm{Pbl} 3$, at $298 \mathrm{~K}$. The Journal of Physical Chemistry Letters 2015, 6, 3209-3212.

(50) Steele, J. A.; Jin, H.; Dovgaliuk, I.; Berger, R. F.; Braeckevelt, T.; Yuan, H.; Martin, C.; Solano, E.; Lejaeghere, K.; Rogge, S. M. J.; Notebaert, C.; Vandezande, W.; Janssen, K. P. F.; Goderis, B.; Debroye, E.; Wang, Y.-K.; Dong, Y.; Ma, D.; Saidaminov, M.; Tan, H.; Lu, Z.; Dyadkin, V.; Chernyshov, D.; Van Speybroeck, V.; Sargent, E. H.; Hofkens, J.; Roeffaers, M. B. J. Thermal unequilibrium of strained black $C s P b \mid<s u b>3</$ sub $>$ thin films. Science 2019, 365, 679-684.

(51) Fu, Y.; Rea, M. T.; Chen, J.; Morrow, D. J.; Hautzinger, M. P.; Zhao, Y.; Pan, D.; Manger, L. H.; Wright, J. C.; Goldsmith, R. H.; Jin, S. Selective Stabilization and Photophysical Properties of Metastable Perovskite Polymorphs of CsPbl3 in Thin Films. Chemistry of Materials 2017, 29, 8385-8394.

(52) Ma, S.; Kim, S. H.; Jeong, B.; Kwon, H.-C.; Yun, S.-C.; Jang, G.; Yang, H.; Park, C.; Lee, D.; Moon, J. Strain-Mediated Phase Stabilization: A New Strategy for Ultrastable $\alpha$-CsPbl3 Perovskite by Nanoconfined Growth. Small 2019, 15, 1900219.

(53) Beygi, H.; Sajjadi, S. A.; Babakhani, A.; Young, J. F.; van Veggel, F. C. J. M. Surface chemistry of assynthesized and air-oxidized PbS quantum dots. Applied Surface Science 2018, 457, 1-10.

(54) Nath, D.; Singh, F.; Das, R. X-ray diffraction analysis by Williamson-Hall, Halder-Wagner and sizestrain plot methods of CdSe nanoparticles- a comparative study. Materials Chemistry and Physics 2020, 239, 122021. 
(55) Zheng, X.; Wu, C.; Jha, S. K.; Li, Z.; Zhu, K.; Priya, S. Improved Phase Stability of Formamidinium Lead Triiodide Perovskite by Strain Relaxation. ACS Energy Letters 2016, 1, 1014-1020.

(56) Schliehe, C.; Juarez, B. H.; Pelletier, M.; Jander, S.; Greshnykh, D.; Nagel, M.; Meyer, A.; Foerster, S.; Kornowski, A.; Klinke, C.; Weller, H. Ultrathin PbS Sheets by Two-Dimensional Oriented Attachment. Science 2010, 329, 550-553.

(57) Méndez, P. F.; Muhammed, S. K. M.; Barea, E. M.; Masi, S.; Mora-Seró, I. Analysis of the UVOzone-Treated SnO2 Electron Transporting Layer in Planar Perovskite Solar Cells for High Performance and Reduced Hysteresis. Solar RRL 2019, 3, 1900191.

(58) Lee, J.-W.; Seol, D.-J.; Cho, A.-N.; Park, N.-G. High-Efficiency Perovskite Solar Cells Based on the Black Polymorph of HC(NH2)2Pbl3. Advanced Materials 2014, 26, 4991-4998.

\section{TOC Figure}

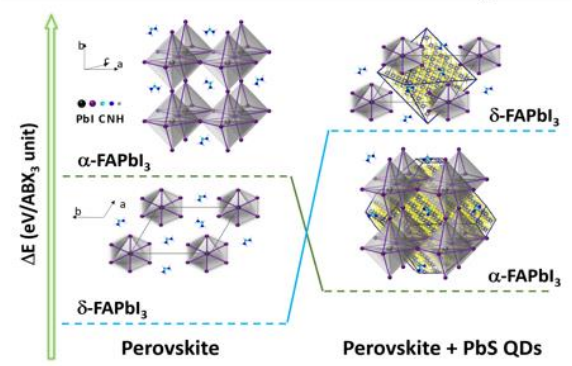

